\title{
A REVIEW OF PUBLISHED DATA ON ANTIMICROBIAL RESISTANCE IN PNEUMOCOCCI ACROSS NORTHERN EUROPE AND RELATIONSHIP TO ANTIBIOTIC CONSUMPTION AND USE OF PNEUMOCOCCAL CONJUGATE VACCINES
}

J. Campling', H. Madhava', A. Vyse', C. Czudek', G. Ellsbury', M. Slack²

1. Pfizer Ltd, Surrey, UK; 2. School of Medicine, Griffith University, QLD, Australia

\section{BACKGROUND}

The most common reason for prescribing antibiotics to children is otitis media Antimicrobial consumption drives antimicrobial resistance

Vaccination leads to reductions in antimicrobial use, which can result in declining rates of antimicrobial resistance

Antimicrobial resistance rates vary considerably across Europe with northern European countries maintaining relatively low rates

\section{OBJECTIVE}

Review available data on antimicrobial resistance, antimicrobial consumptio and relevant interventions across northern Europe

\section{METHODS}

Publications were identified through Medline and Embase searches, limited to human, English language and the last 10 years

Search terms included "Pneumococcus OR Streptococcus pneumoniae OR Pneumococci AND Antimicrobial Resistance OR AMR OR penicillin OR erythromycin OR tetracycline OR macrolides AND UK OR Ireland OR Denmark OR Sweden OR Norway OR Finland OR Latvia OR Lithuania OR Estonia OR Netherlands OR Iceland OR Greenland"

We also reviewed data from the European Surveillance for Antimicrobial Consumption Network (ESAC-Net) ${ }^{1}$ and the European Antimicrobial Resistance Surveillance Network (EARS-Net) ${ }^{2}$

\section{RESULTS}

210 articles were identified and of those we deemed 95 warranted further review based upon title (TABLE 1)

" 45 of the 95 papers were concerned with pneumococcal infections in children. 18 reported

None of the papers examined the impact of PCVs on antimicrobial
consumption

- In 2016, EARS-Net reported pneumococcal penicillin resistance rates across northern Europe ranged from 2.2\% (The Netherlands) to $16.5 \%$ (Ireland) - Macrolide resistance rates ranged from $0 \%$ (Iceland) to $18.1 \%$ (Lithuania). In the same year ESAC-Net reported consumption of antibacterials in the community ranged from 10.4 (The Netherlands) to 21.0 (Iceland) defined daily dose per 1,000 inhabitants per day

All northern European countries recommend pneumococcal conjugate vaccine (PCV) immunisation in children
RESULTS (CONT)

TABLE 1. COUNTRY OF ORIGIN OF IDENTIFIED MANUSCRIPTS

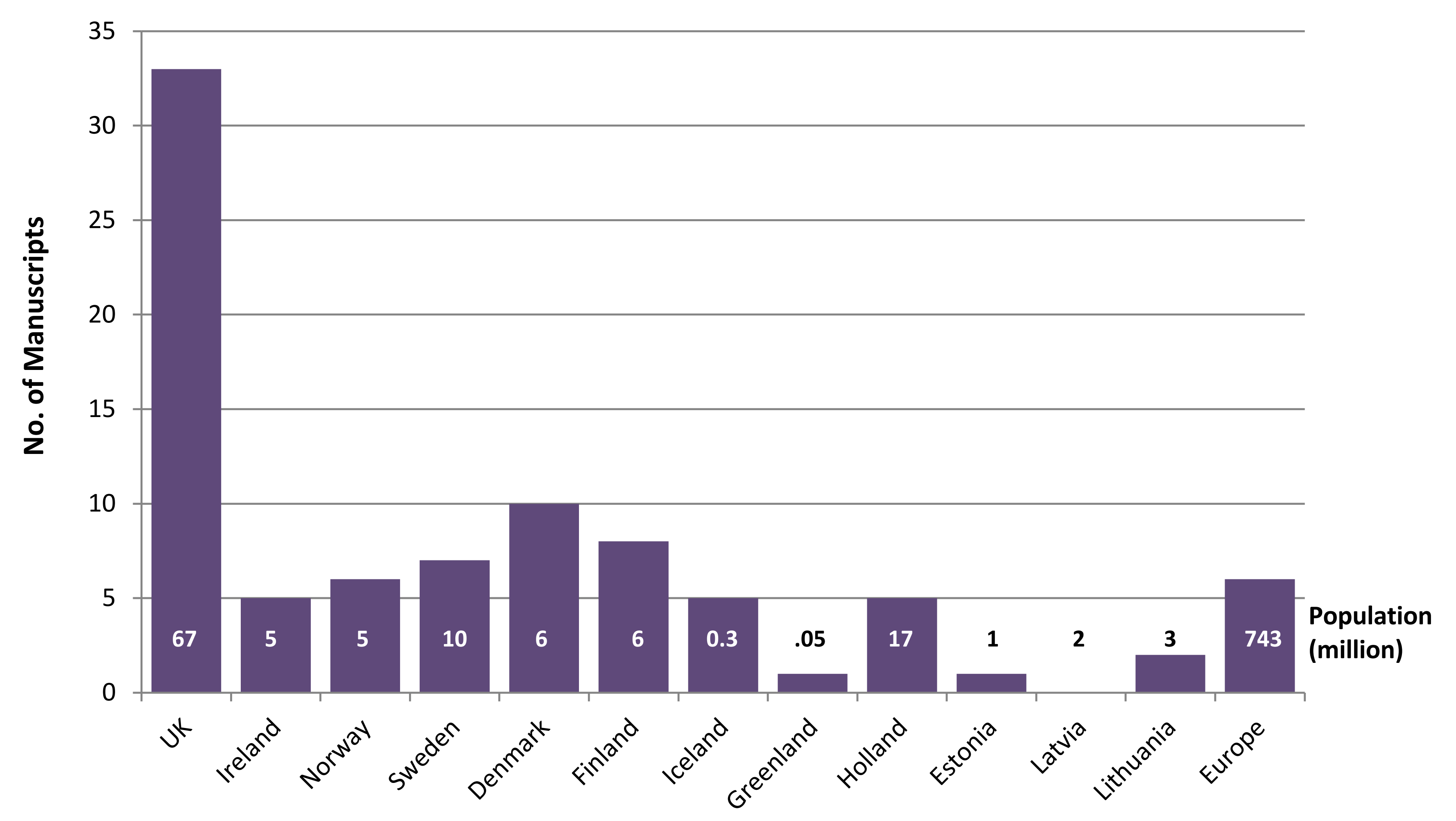

The aim of antibiotic stewardship programmes is to optimise antibiotic usage and combat antimicrobial resistance. The impact of paediatric immunization programmes on antibiotic consumption and antimicrobial resistance should also be considered

More than $90 \%$ of antimicrobials prescribed in Europe are for patients in the community ${ }^{3}$

Non-invasive pneumococcal infections (especially acute otitis media [AOM]) are one of the main reasons for antimicrobial prescribing in the Western World ${ }^{4}$

In Iceland, prior to the introduction of PCV immunisation, children aged $<7$ years consumed $20 \%$ of all antimicrobials prescribed ${ }^{4}$

In addition, $>50 \%$ of prescriptions issued were to treat $\mathrm{AOM}^{4}$

- Bell and colleagues in a review of over 200 papers reported that increased consumption of antibiotics in the community may not only produce greater resistance at the individual patient level but may also produce greater resistance at the community, country, and regional levels

Antimicrobial prescriptions are likely one of the main causes of the emergence of antimicrobial-resistant organisms in the community

\section{RESULTS (CONT)}

- Interventions to reduce antimicrobial prescribing in Southern Sweden resulted in a $52 \%$ reduction in antimicrobial prescribing in children but no decline in the proportion of penicillin-nonsusceptible Streptococcus pneumoniae (PNSP) $\left(\mathrm{MIC}_{\text {penG }} \geq 0.12 \mathrm{mg} / \mathrm{L}\right)$

- A study in Sweden ${ }^{8}$ examined all penicillin-resistant Streptococcus pneumoniae (PRP) $\left(\mathrm{MIC}_{\mathrm{penG}} \geq 0.5 \mathrm{mg} / \mathrm{L}\right)$ isolated between 1997 and 2003 and found little correlation between antibiotic sales and PRP indicating

other factors important in controlling PRP

The major serogroups isolated from children were $9 \mathrm{~V}(51 \%), 14(29 \%), 23$ and 6 (all serotypes/serogroups included in PCV7)

This study provides evidence that other strategies to control antimicrobial resistance are required

- In Iceland in 1992 antimicrobial consumption declined in children but the rate of PNSP was only very modestly reduced (8.6\% to $8.0 \%)$ and in some communities PNSP clones actually increased

All of these studies were conducted before national infant immunisation with PCVs was introduced

\section{CONCLUSIONS}

Antimicrobial stewardship has an important role to play in reducing unnecessary antimicrobial prescriptions which is a factor in the developmen of antimicrobial resistance

However, evidence from Northern Europe clearly demonstrates that other factors are of importance, including the use of vaccines to reduce the number of infections (notably upper respiratory tract infections) which result in antimicrobial prescriptions

PCVs provide evidence that vaccines can play an important role in reducing rates of antimicrobial resistance, if the vaccine targets resistant serotypes

The Joint Committee on Vaccination \& Immunisation in the UK stated immunisation is an important strategy for tackling AMR and recommended future reviews of vaccination programmes should include the potential benefits of vaccines in reducing antimicrobial use

We encourage other national immunisation technical advisory technica committees to follow this policy

\section{REFERENCES}

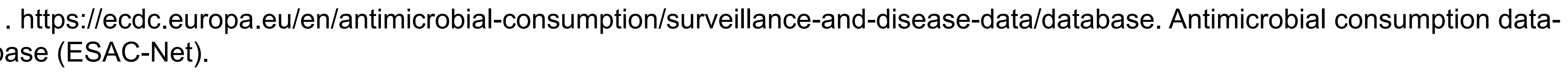

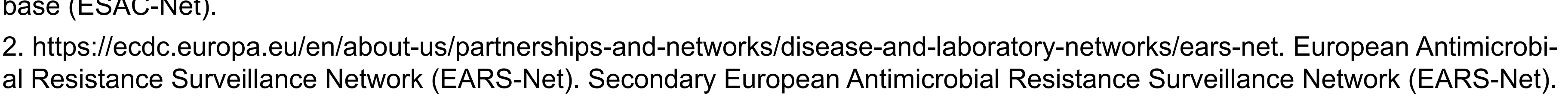

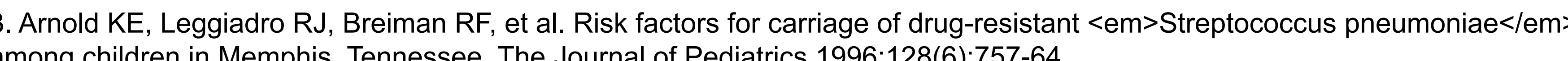

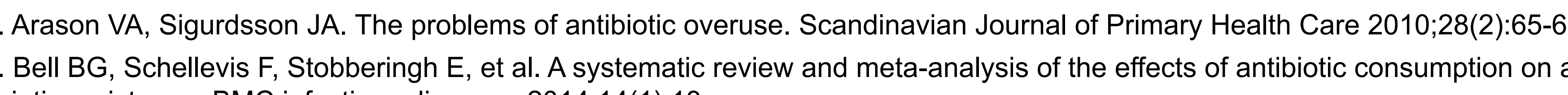

6. Cars O, Hogberg LD, Murray M, et al. Meeting the challenge of antibiotic resistance. BMJ (Clinical research ed)

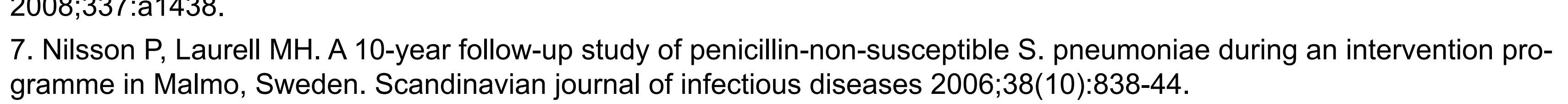

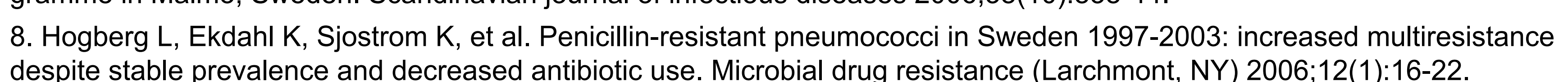
9. http://wwww worddometers decreased antibion cis 